subtypes, immunohistology, and DNA ploidy. Am J Surg Pathol 1995; 19: 798-809.

3. O'Malley DP, George TI, Orazi A, et al. Benign and Reactive Conditions of Lymph Node and Spleen. Atlas of Nontumor Pathology. Washington, DC: American Registry of Pathology, 2009.

4. Chen P, Lin JJ, Lu CS, et al. Carbamazepine-induced toxic effects and HLA-B*1502 screening in Taiwan. N Engl J Med 2011; 364: 1126-33.

5. Fleming P, Marik PE. The DRESS syndrome: the great clinical mimicker. Pharmacotherapy 2011;31: 332

6. De A, Rajagopalan M, Sarda A, et al. Drug reaction with eosinophilia and systemic symptoms: an update and review of recent literature. Indian J Dermatol 2018; 63: 30-40.

7. Husain Z, Reddy BY, Schwartz RA. DRESS syndrome: Part I. Clinical perspectives. J Am Acad Dermatol 2013; 68. 693e1-14; 706-8.

8. Cumbo-Nacheli G, Weinberger J, Alkhalil M, et al. Anticonvulsant hypersensitivity syndrome: is there a role for immunomodulation? Epilepsia 2008; 49: 2108-12.

9. Abbondazo SL, Irey NS, Frizzera G. Dilantin-associated lymphade nopathy. Spectrum of histopathologic patterns. Am J Surg Pathol 1995 19: $675-86$.

10. Ovallath S, Remya RK, Kumar C, et al. Granulomatous lymphadenopathy secondary to phenytoin therapy. Seizure 2013; 22: 240-1.

11. Lapes MJ, Vivacqua RJ, Antoniades K. Immunoblactic lymphadenop athy associated with phenytoin (diphenylhydantoin). Lancet 1976; 1 : 198.

12. Subbannan K, Gujral JS. Necrotizing lymphadenitis associated with the phenytoin-induced hypersensitivity syndrome. South Med J 2005; 98: 937-9.

13. Ganga A, Corda D, Gallo Carrabba G, et al. A case of carbamazepineinduced lymphadenopathy resembling Kikuchi disease. Eur Neurol 1998: 39: 247-8.

DOI: https://doi.org/10.1016/j.pathol.2019.04.010

\section{Is secretory breast carcinoma underdiagnosed? In the era of targeted therapy should there be a low threshold to screen for NTRK immunohistochemistry in triple negative breast cancers?}

Sir,

Secretory breast carcinomas are reported to be very rare, accounting for $<0.1 \%$ cases of invasive breast cancers, but may be under-recognised. ${ }^{1}$ Initially termed juvenile breast carcinoma', ${ }^{2}$ secretory breast carcinomas are characteristically driven by oncogenic ETV6-NTRK3 gene fusions which are extremely rare in all other breast carcinomas. ${ }^{1}$ The neurotrophic tyrosine receptor kinase (NTRK) group of genes, NTRK1, NTRK2 and NTRK3, encode for a family of receptor tyrosine kinases $\operatorname{Trk} \mathrm{A}, \operatorname{TrkB}$, and $\operatorname{TrkC}$, respectively. ${ }^{3,4}$ These receptors normally contribute to neuronal development, function, survival and proliferation. ${ }^{3,4}$ In addition to secretory carcinoma of the breast, uncontrolled activation of the NTRK genes, usually due to fusion events, have proven to be the most common driver of some relatively rare tumours (including infantile fibrosarcoma and secretory carcinoma of the salivary gland) but are also found at a very low incidence in a diverse range of malignancies. ${ }^{3-5}$

$N T R K$ gene fusions are becoming increasingly important to identify in the routine clinical laboratory because of the availability of rationally designed targeted therapies including the specific NTRK inhibitor larotrectinib. ${ }^{3-5}$ Larotrectinib has demonstrated efficacy against a wide variety of tumours harbouring activating NTRK fusions and is now US Food and Drug Administration approved.
However, the optimal strategy for identifying NTRK fusions in the routine clinical setting is still unclear. While screening immunohistochemistry has been suggested to have a potential role in triaging molecular testing, it is not completely sensitive or specific and expensive molecular methods remain the gold standard. ${ }^{6}$ Whilst it would be beyond the resources of most pathology laboratories to screen all malignancies with a low risk for NTRK fusions (for example all breast carcinomas) with molecular techniques, it may be reasonable to justify the cost for specific tumours with a high incidence of NTRK fusions (for example secretory carcinomas or infantile fibrosarcomas). Despite questions about its sensitivity and specificity, it may be reasonable to perform screening NTRK immunohistochemistry to triage molecular testing on tumours with a low or intermediate risk of harbouring NTRK fusions.

Typically secretory breast carcinomas lack oestrogen receptor, progesterone receptor and human epidermal growth factor (HER2) expression, and therefore can be considered a specific example of triple negative breast cancer (TNBC). ${ }^{4}$ That is, the subgroup of all breast carcinomas classified as TNBC is enriched for secretory carcinoma and may have a higher incidence of NTRK gene rearrangements, particularly if secretory carcinoma is under-recognised. Therefore, we postulated that there may be a role for NTRK immunohistochemistry in all TNBCs to triage molecular testing. Indeed there are case reports of tumours initially thought to be TNBC being re-classified as secretory carcinoma upon review and this having a significant impact on treatment.

We sought to assess whether screening immunohistochemistry for NTRK may have a role in the routine assessment of TNBC in order to triage molecular testing, by performing NTRK immunohistochemistry in a large number of TNBCs with follow up molecular testing on all cases with some positive staining.

A tissue microarray (TMA) of consecutive primary triple negative breast carcinomas was used. The TMA included two $1 \mathrm{~mm}$ cores from all triple negative breast carcinomas that had undergone resection between 2005 and 2015 at our institution. The clinical characteristics and androgen receptor status of this cohort have been previously reported. ${ }^{8} \mathrm{We}$ performed pan-NTRK immunohistochemistry using a commercially available rabbit monoclonal antibody (clone EPR17341; Abcam, USA; dilution 1/50, after heat induced antigen retrieval in an alkaline retrieval solution) using similar methods to that reported by others.

The TMA sections stained with NTRK were scored by two independent observers who were blinded to all clinical and pathological details. NTRK IHC was repeated on whole sections from all cases with any positive staining identified by one or both observers.

There were 137 cases in this TMA cohort, of which 11 (8\%) demonstrated some positive staining on TMAs. All had at least some confirmed positive expression when the scoring was repeated on whole sections. The tumour and patient characteristics of the 11 positive cases are presented in Table 1. Briefly, the ages ranged from 45 to 83 years (mean 59.5). There was one case initially diagnosed as secretory breast carcinoma (the only secretory carcinoma diagnosed at our institution over the 11 year period). Eight cases were originally classified as invasive ductal carcinomas of no special type and two as invasive ductal carcinomas with medullary features. 
Table 1 The clinical and pathological features of triple negative breast cancers demonstrating any positive staining for NTRK by immunohistochemistry

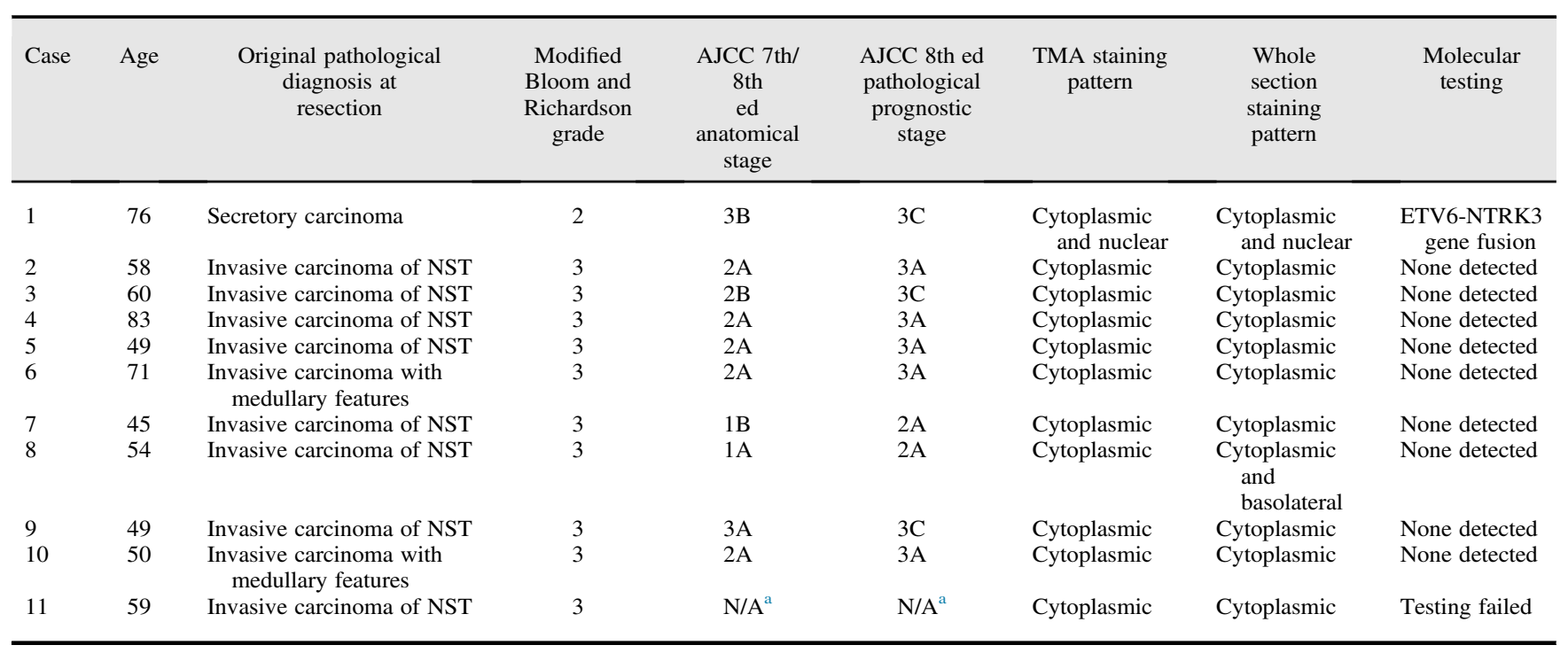

AJCC, American Joint Committee on Cancer; NST, no special type; TMA, tissue microarray.

${ }^{a}$ pNX: regional lymph nodes cannot be assessed (not removed for pathological study).

The known secretory breast carcinoma displayed diffuse weak to moderate cytoplasmic and nuclear positivity confirmed on whole section (Fig. 1). Ten other cases displayed variable staining, including one with both cytoplasmic and basolateral staining and some cases with both tumour localised and non-specific background staining (Table 1, Fig. 1). All cases which displayed at least focal cytoplasmic and/or nuclear staining were selected for molecular testing.

Paraffin blocks from all 11 cases were analysed on a fee for service basis with the GeneTrails Solid Tumour Fusion Gene panel at Knight Diagnostics, Portland, Ohio, USA. This targeted RNA-based panel utilises next generation sequencing tested for 20 targeted fusion genes: NTRK1, NTRK2, NTRK3, AKT3, ALK, BRAF, CD74, EGFR EML4, ERBB4, ERG, ETV6, FGFR1, FGFR2, FGFR3, MET, NOTCH1, NOTCH2, NRG1, NUTM1, PDGFRA, RAF1, RET and ROS1 (see Beadling et al. ${ }^{9}$ for full details).

Of the 11 cases undergoing molecular testing, only one case harboured a confirmed NTRK fusion. The fusion positive case was the single prospectively identified secretory carcinoma. Testing of one case failed, whereas the remaining nine cases were negative for NTRK fusions. That is, the addition of screening immunohistochemistry for NTRK did not result in

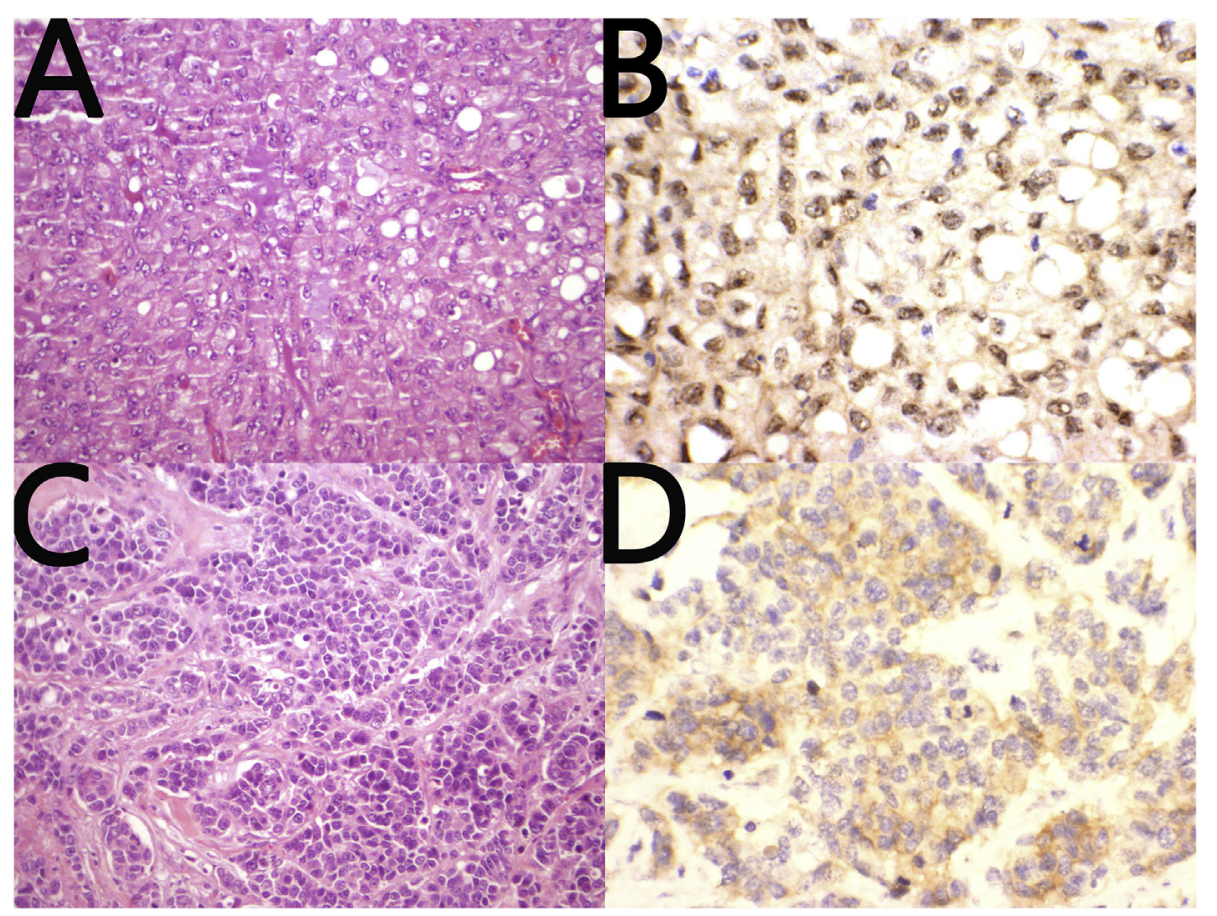

Fig. 1 Serial H\&E (A,C) and NTRK immunohistochemistry (B,D) stained sections from the known secretory carcinoma (A,B) demonstrated to harbour the ETV6NTRK 3 fusion and a triple negative breast carcinoma (C,D) demonstrated to lack the fusion. 
any more TNBCs harbouring NTRK gene rearrangements being identified than would have been found if testing was limited to the case classified as secretory carcinoma based on morphological findings alone.

We accept that there are some limitations to this study including its retrospective nature and, most importantly, that it was TMA based and may have missed some cases with focal expression. We also note that molecular testing was not performed on all TNBCs and the study was not designed or intended to test the sensitivity of NTRK immunohistochemistry in TNBCs.

Despite these limitations, on the basis of this study we conclude that reflex screening immunohistochemistry of all TNBCs using the rabbit monoclonal antibody EPR17341 is a very low yield approach to identifying breast carcinomas harbouring NTRK gene rearrangements and is difficult to justify in the routine clinical setting. Given the non-specific staining demonstrated by this study, we doubt that NTRK immunohistochemistry will be useful in clinical practice to confirm a morphological suspicion of secretory carcinoma. Furthermore, if targeted therapy is being considered for a patient with a potential diagnosis of secretory carcinoma, we would recommend testing for NTRK gene rearrangements by molecular means rather than using the surrogate marker of NTRK immunohistochemistry, given its apparent lack of specificity in this setting.

Conflicts of interest and sources of funding: The authors state that there are no conflicts of interest to disclose.

\section{Matthew Zaborowski ${ }^{1,2}$, Anthony J. Gill ${ }^{1,2,3}$ \\ ${ }^{1}$ Cancer Diagnosis and Pathology Group, Kolling Institute of Medical Research, Royal North Shore Hospital, St Leonards, NSW, Australia; ${ }^{2}$ NSW Health Pathology, Department of Anatomical Pathology, Royal North Shore Hospital, Sydney, NSW, Australia; ${ }^{3}$ University of Sydney, Sydney, NSW, Australia}

Contact Anthony J Gill.

E-mail: affgill@med.usyd.edu.au

1. Lee SG, Jung SP, Lee HY, et al. Secretory breast carcinoma: a report of three cases and a review of the literature. Oncol Lett 2014; 8: 683-6.

2. McDivott R, Stewart FW. Breast carcinoma in children. JAMA 1966; 195 : 388-90.

3. Cocco R, Scaltriti M, Drilon A. NTRK fusion-positive cancers and TRK inhibitory therapy. Nat Rev Clin Oncol 2018; 15: 731-47.

4. Lange AM, Lo HW. Inhibiting TRK proteins in clinical cancer therapy. Cancers (Basel) 2018; 10: 105.

5. Drilon A, Laetsch TW, Kumar S, et al. Efficacy of larotrectinib in TRK fusion-positive cancers in adults and children. N Engl J Med 2018; 378: 731-9.

6. Hechtman JF, Benayed R, Hyman DM, et al. Pan-Trk immunohistochemistry is an efficient and reliable screen for the detection of NTRK fusions. Am J Surg Pathol 2017; 41: 1547-51.

7. Benabu JC, Stoll F, Koch A, et al. De-escalating systemic therapy in triple negative breast cancer: the example of secretory carcinoma J Gynecol Obstet Hum Reprod 2018; 4: 163-5.

8. Zaborowski M, Pearson A, Sioson L, et al. Androgen receptor immunoexpression in triple-negative breast cancers: is it a prognostic factor? Pathology 2019; 51: 327-9.

9. Beadling C, Wald AI, Warrick A, et al. A multiplexed amplicon approach for detecting gene fusions by next-generation sequencing. J Mol Diagn 2016; 18: 165-75.

DOI: https://doi.org/10.1016/j.pathol.2019.04.012

\section{Lack of clinical activity with crizotinib in a patient with FUS rearranged rhabdomyosarcoma with ALK protein overexpression}

Sir,

We have previously reported on a 23-year-old male presenting with a recently described novel subtype of rhabdomyosarcoma (RMS) with a defining FUS rearrangement. ${ }^{1}$ This entity, first described in $2018,{ }^{2}$ carries the unusual combination of myogenin, MyoD1 and ALK protein overexpression in combination with a FUS/EWSR1-TFCP2 fusion portending an aggressive clinical course. Strong cytoplasmic staining for ALK1 (ALK 01 clone) was identified on immunohistochemistry and ALK (2p23) disruption was identified in $80 \%$ of cells with loss of $5^{\prime}$ signal on fluorescence in situ hybridisation (FISH). Next generation sequencing confirmed a large ALK deletion of exons 2-16 (TruSight Tumour 170 panel; Illumina, USA).

Clinically, this patient had rapidly progressed through five cycles of anthracycline based chemotherapy in conjunction with definitive external beam radiation to his primary sinonasal RMS. In light of the immunohistochemical (IHC) and FISH findings, and in the setting of his aggressive clinical course and chemoresistance, a decision was made with the family to self-fund crizotinib (Xalkori, PF-02341066, Pfizer) at a dose of $250 \mathrm{mg}$ twice daily.

Treatment was delivered between August and September 2018. Treatment was tolerated without adverse events. Early radiological assessment was conducted after 4 weeks of therapy with evidence of stable disease in the primary sinonasal RMS but clear metabolic and morphological progression of his bilateral pleuro-pulmonary disease (Fig. 1A,B, pre crizotinib delivery; Fig. 1C, after 1 month of crizotinib). The patient was subsequently admitted for management of his symptomatic pleural effusion and unfortunately died 3 weeks later.

This case highlights the lack of clinical activity seen in the described patient in targeting ALK, which may have potential implications for other RMS patients with similar IHC, FISH and molecular correlates. The clinical activity appears contrary to other tumour subtypes where some magnitude of response in those with $5^{\prime}$ deletion has been reported, albeit to lesser degree than traditional $3^{\prime}$ and $5^{\prime}$ split signals. ${ }^{3}$ In our case, the ALK over-expression on IHC was hypothesised to represent a truncated isoform in the presence of a large ALK gene deletion without the presence of the extracellular domain. This pattern of ALK exon 2-17 deletion has been described in anaplastic large cell lymphoma ${ }^{4}$ and neuroblastoma ${ }^{5}$ leading to a truncated isoform that lacked most of the extracellular domain encompassing the LDLa domain, MAM domains, and a glycine-rich region. ALK expression by IHC using the ALK1 antibody binds within the tyrosine kinase domain at the c-terminal end of the $A L K$ gene (exon 20-29), supporting the strong intracytoplasmic ALK protein expression observed. It is possible that ALK protein expression alone does not constitute a valid target in RMS, and that what is being demonstrated is a passenger effect, ${ }^{6,7}$ with further research required to understand the mechanisms underlying this. Alternatively, more potent signalling inhibition such as that achieved with newer generation ALK inhibitors may be required to achieve a clinically meaningful response. 